\title{
Frequency-dependent signal transmission and modulation by neuromodulators
}

\author{
Hiroshi T. Ito ${ }^{1}$ and Erin M. Schuman ${ }^{1,2 *}$ \\ 1 Division of Biology, California Institute of Technology, Pasadena, CA, USA \\ 2 Howard Hughes Medical Institute, Pasadena, CA, USA
}

The brain uses a strategy of labor division, which may allow it to accomplish more elaborate and complicated tasks, but in turn, imposes a requirement for central control to integrate information among different brain areas. Anatomically, the divergence of long-range neuromodulator projections appears well-suited to coordinate communication between brain areas. Oscillatory brain activity is a prominent feature of neural transmission. Thus, the ability of neuromodulators to modulate signal transmission in a frequency-dependent manner adds an additional level of regulation. Here, we review the significance of frequency-dependent signal modulation in brain function and how a neuronal network can possess such properties. We also describe how a neuromodulator, dopamine, changes frequency-dependent signal transmission, controlling information flow from the entorhinal cortex to the hippocampus.

Keywords: neuromodulator, frequency-dependent modulation, oscillation, dopamine, CA1

Edited by:

Rafael Yuste, Columbia University, USA

Reviewed by:

Charles J. Wilson, University of Texas

at San Antonio, USA

Miles A. Whittington, Newcastle

University, UK

* Correspondence:

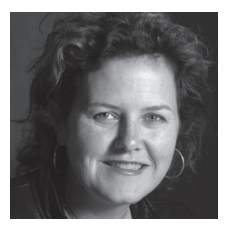

Erin M. Schuman obtained her B.A. from the University of Southern California and her Ph.D. from Princeton University. Her laboratory is interested in synaptic transmission and plasticity. schumane@caltech.edu

\section{NEUROMODULATORS CONTROL REGIONAL INTERACTIONS IN THE BRAIN}

A number of studies suggest that the brain exhibits functional localization, i.e. each brain region has a somewhat specialized role (Boling et al., 2002; Passingham et al., 2002). Most animal behaviors require the coordinated activity of sensory, integrative and motor brain areas. As the sensory landscape undergoes change, the interaction among brain areas should also be dynamic and change depending on the situation. For example, when you meet a new person, initially you may try to memorize his or her face (encoding information), but the next time you see this person, you will recognize his or her face and may remember several events associated with this person (retrieving information). Given that the synaptic transmission between neurons is a basic unit of information processing, it is crucial to understand how synaptic modulation can change interactions among brain areas.

Neurotransmitters, such as dopamine, norepinephrine, serotonin, or acetylcholine, play an important role in state-dependent modulation of the brain (Kodama et al., 2002; Robbins, 2005; Takakusaki et al., 2006). These neurotransmitters, often called neuromodulators, are synthesized and released from a relatively small number of specialized neurons, which are primarily located in several distinct nuclei in the basal forebrain, midbrain or brainstem (Siegel et al., 1999). Through long-range connections these neuromodulatorreleasing neurons make synaptic contacts with many different brain areas. Neuromodulators released from synaptic terminals are also capable of diffusing over substantial distances $(>10 \mu \mathrm{m})$ and can act on receptors remote from release sites (volume transmission; Venton et al., 2003; Zoli et al., 1998). Thus, at the apparent cost of spatial selectivity, the information from neuromodulator-releasing neurons can be broadcast to a large area of the brain. As such, activity changes in a small number of neurons can exert a broadcast influence on many brain areas, coordinating a functional change across areas (Hasselmo, 1995). 
Memory encoding

A process whereby external information

(e.g. object shape/color, spatial

locations, smells, sounds etc.) is

converted to a representation of multiple neuronal activities. This is the first step for the memory formation.

The remaining two steps are memory storage and retrieval.

Neuromodulator

A class of neurotransmitters that can diffuse over long distances and

influence multiple neurons and synapses. Most neuromodulators activate G-protein coupled receptor and alter excitatory and/or inhibitory synaptic transmission or ionic conductances. Examples of neuromodulators include dopamine, norepinephrine, acetylcholine, serotonin or histamine.

\section{Oscillatory activities}

Repetitive temporal variation of voltage signals observed at different frequencies in electroencephalography, extracellular or intracellular recordings in the brain. Multiple oscillatory frequencies can be recorded ranging from 0.05 to $500 \mathrm{~Hz}$, each of which appears to be independently controlled. For example, the rat hippocampus generates theta (4-12 Hz), gamma $(40-100 \mathrm{~Hz})$ and fast oscillations (140-200 Hz) independently.

Nonlinear signal transduction A process whereby a change in input signal does not result in a correspondingly similar degree of change in output signals. Or, a process where an output signal generated by multiple inputs is not a simple sum of the inputs.

\section{FREQUENCY-DEPENDENT SIGNAL MODULATION AND OSCILLATORY ACTIVITIES}

In electroencephalograms or local field potential recordings, the brain activities are observed as multiple oscillators at different frequencies. A number of studies have described apparent links between specific oscillatory activities and particular brain functions (Buzsaki and Draguhn, 2004; Osipova et al., 2006; Palva and Palva, 2007). These oscillatory activities are not just epiphenomena, but the brain appears to utilize them for information coding, for example, to bind distributed information in the cortex (Engel et al., 2001; Varela et al., 2001) or to select phase-locked activities (Laurent, 2002). These results imply that oscillatory activities may play an important role in regulating information flow in the brain. Thus, it is worthwhile to assess how a neuronal network will respond to different frequency stimulation.

In monosynaptic transmission, the magnitude of the postsynaptic response evoked by presynaptic stimulation is intrinsically dependent on stimulation frequency (Markram et al., 1998). For example, during the delivery of multiple stimuli at close time intervals, the size of postsynaptic potentials can become larger or smaller, phenomena known as paired-pulse facilitation or depression, respectively (Zucker and Regehr, 2002) (Figure 1). Both presynaptic and postsynaptic mechanisms have been implicated in these processes. For example, changes in neurotransmitter release probability, availability of readily releasable pool of synaptic vesicles (Dobrunz and Stevens, 1997), postsynaptic receptor desensitization (Koike-Tani et al., 2008) or surface mobility of postsynaptic receptors (Heine et al., 2008) have all been proposed to play an important role in frequency-dependent modulation of synaptic transmission.

Differences in neuronal morphology and molecular composition will further extend possible patterns of frequency dependency modulation in signal transmission. The brain is composed of hundreds of types of neurons and each has distinct morphology, channel/receptor density and distribution, which determines differences in threshold for action potentials, firing patterns or synaptic plasticity (Mainen and Sejnowski, 1996; Shepherd, 2004). Therefore, the same input stimuli applied to different types of neurons can expose one type of neuron that is sensitive to low frequency stimulation and another that is sensitive to high frequency stimulation (Buzsaki and Draguhn, 2004; Hutcheon and Yarom, 2000). Frequency-dependent signal transmission by distinct neuronal types has been observed in the hippocampus (Mori et al., 2004; Pouille and Scanziani, 2004).
Signal gating based on input stimulation patterns can also be generated based on differences between monosynaptic and disynaptic transmission. Disynaptic transmission differs from monosynaptic transmission in two ways: (1) the temporal delayimposed by the additional synapse, and (2) the nonlinear signal transduction with respect to the strength of input stimulation, due to requirement for action potential generation. These differences will play an important role in temporally selective filtering of input signals because the influence of disynaptic inputs are evident when inputs are delivered close in time (Ito and Schuman, 2007) (Figure 1). Thus, a neuronal network does not pass all signals equally, but rather, has a bias for a certain signal pattern. As such, changing oscillatory activities observed in the brain may lead to dynamic modulation of regional interactions.

One effect of neuromodulator receptor activity is the modulation of ion channels and receptors via intracellular signaling, which influences neuronal activity or synaptic transmission (Siegel et al., 1999). An explored area, however, concerns the influence of neuromodulators at a network level. Ito and Schuman (2007) recently examined how the neurotransmitter dopamine can change a frequency-dependent profile of signal transmission at temporoammonic-CA1 synapses in the hippocampus. The primary effect of dopamine at these synapses is presynaptic depression due to a decrease in release probability of synaptic vesicles. Surprisingly, however, this simple form of modulation has a large impact on the neuronal circuit as a whole that is, dopamine depresses low frequency input signals but enhances high frequency signals (Figure 2A). Thus, dopamine imposes a high pass filter on this pathway exerting a preference for certain frequency inputs. This is a good example of how a simple synaptic modulatory action can have a large impact at a network level. In fact, the differences between monosynaptic and disynaptic transmission, as described in the last paragraph, plays an important role in generating this effect (Figure 2B).

\section{FREOUENCY-DEPENDENT MODULATION BY DOPAMINE AT THE TEMPOROAMMONIC PATHWAY IN THE HIPPOCAMPUS}

Dopamine is a neuromodulator, that plays crucial roles in motor control, learning and memory, or addictive behavior formation (Hikosaka, 2007; Wise, 2004). Most dopaminergic neurons are located in two nuclei, the ventral tegmental area and substantia nigra compacta (Bjorklund and Dunnett, 2007). Activation of these neurons during animal learning has been well characterized in monkey. Schultz and colleagues examined the in vivo activities of dopaminergic neurons during 


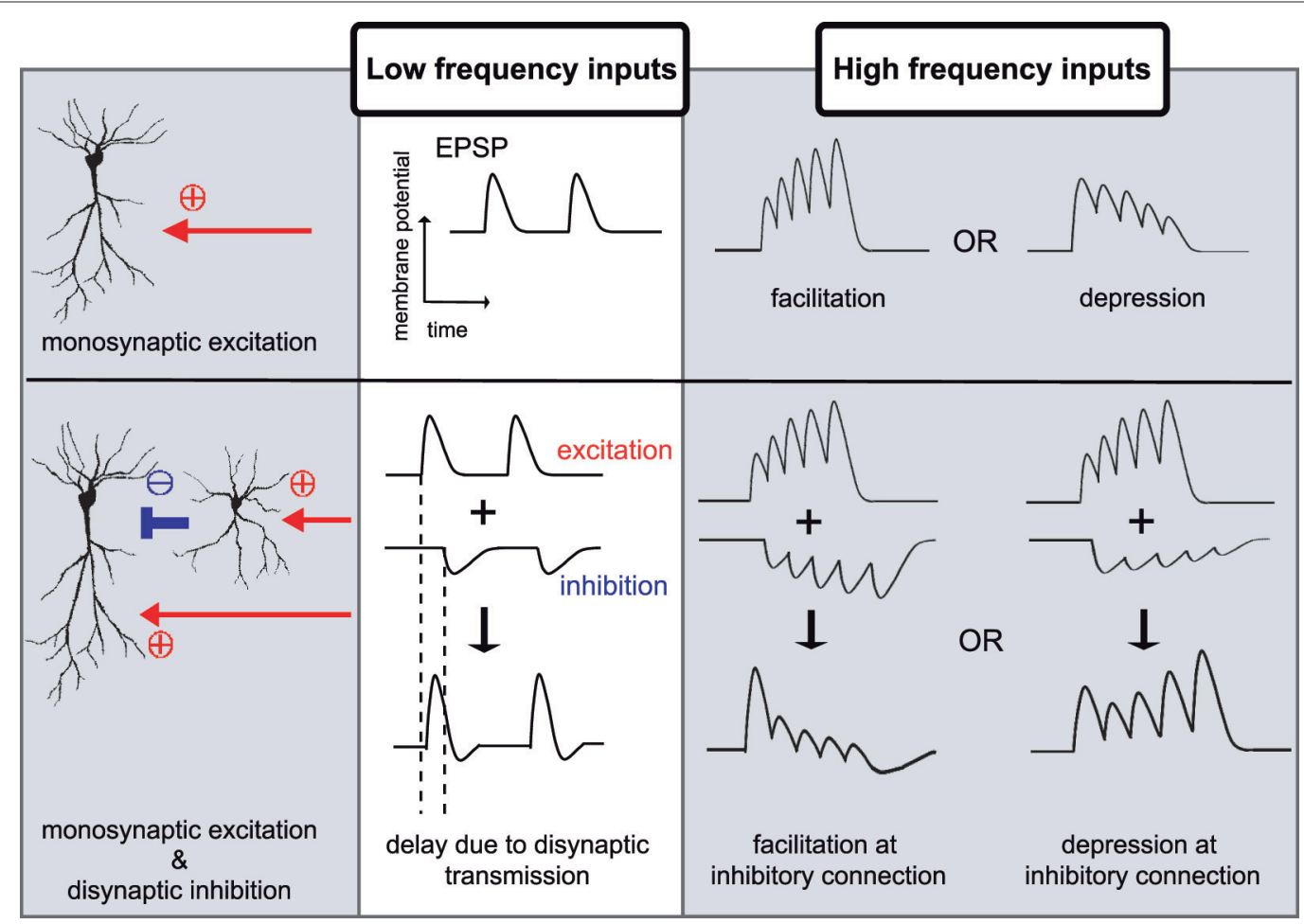

Figure 1 | Examples of differential signal transmission between low and high frequency inputs. In monosynaptic transmission, low frequency inputs elicit constant postsynaptic responses, however, high frequency inputs induce facilitation or depression in signal transmission. A simple network composed of monosynaptic excitation and disynaptic inhibition exhibits more diverse patterns of signal modulations. The influence of disynaptic transmission on monosynaptic transmission is not evident during low frequency inputs, due to the temporal delay imposed by the additional synapse. During high frequency stimulation, however, disynaptic inhibition effectively influences monosynaptic excitation. Because each neuron shows distinct response to high frequency stimuli, disynaptic inhibitory connection shows facilitation, depression, or some characteristic response, which will, together with modulation in monosynaptic excitation, expand differential patterns of signal transmission between low and high frequency stimulation. a conditioning task. In this study, the activity of dopaminergic neurons appears to reflect differences between internal expectations and actual outcomes, i.e. expectation errors (Schultz, 1998). Thus, one function of the dopamine system is to provide information about the salience of environmental stimuli for learning (McClure et al., 2003).

Dopaminergic neurons extend their axons to the hippocampus, predominantly to distal part of area CA1 and the subiculum (Gasbarri et al., 1997). A number of clinical and experimental studies suggest the involvement of the hippocampus in declarative memory formation (Squire et al., 2004). Similar to dopaminergic neurons, the hippocampus also exhibits differential activation during presentation of novel or familiar stimuli (Knight, 1996; Rutishauser et al., 2006). A desirable feature of a learning system is the ability to differentiate novel and familiar information, because it enables the system to "know" how synaptic connections should be modified based on input signals (Blumenfeld et al., 2006). In turn, the hippocampus influences the dopamine system as well, forming a functional loop (Lisman and
Grace, 2005). Thus, the interaction between the dopamine system and the hippocampus may be a key to understand the neural circuits that subserve learning. Indeed, several studies suggest the involvement of dopamine in the hippocampal dependent learning. For example, dopamine D1 receptor knockout mice exhibit a learning deficit in the Morris water maze task, which requires hippocampal function (El-Ghundi et al., 1999). In addition, when 6-hydroxy dopamine was injected to selectively disrupt dopaminergic axons to the hippocampus, these animals also showed a deficit in the Morris water maze task (Gasbarri et al., 1996). These data indicate that it is important to assess how dopamine will influence information flow in the hippocampal circuit.

As described by Andersen et al. (1969), the hippocampal network is a feedforward circuit composed of three primary synaptic connections. Compared with other cortical circuits, the hippocampal circuit is unique in that these connections are largely unidirectional (Witter and Amaral, 2004). Area CA1 in the hippocampus receives excitatory inputs from area $\mathrm{CA} 3$, via 

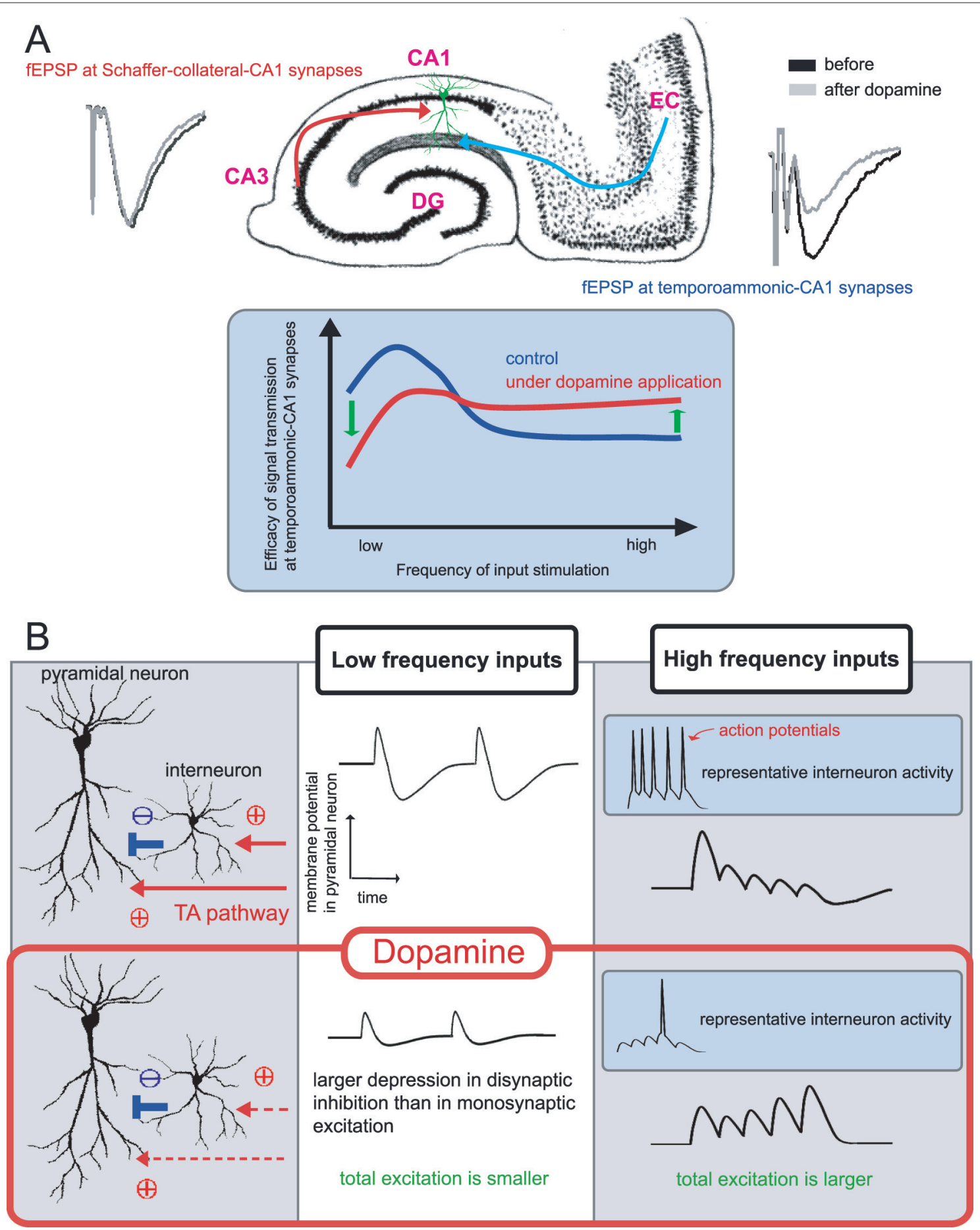

Figure 2 | Frequency dependent modulation of dopamine at temporoammonic-CA1 synapses. (A) Area CA1 in the hippocampus receives two distinct excitatory inputs, one is from area CA3 (the Schaffer-collateral pathway) and another is from the entorhinal cortex (the temporoammonic pathway). Dopamine selectively depresses baseline field EPSP at temporoammonic-CA1 synapses, and has no significant effect on Schaffercollateral-CA1 synapses. Under the delivery of different frequency stimuli, dopamine exerts a frequency-dependent modulation at temporoammonic-CA1 synapses, depressing low-frequency signals but enhancing high-frequency signals. (B) Primary effect of dopamine is a depression of excitatory synapses onto both pyramidal neurons and interneurons. The influence of disynaptic inhibition on monosynaptic excitation is not evident during low frequency inputs, thus dopamine simply depresses total excitation to pyramidal neurons. In contrast, during high frequency stimulation, disynaptic inhibition becomes influential and intrinsically strong inhibitory connections at temporoammonic-CA1 synapses suppress monosynaptic excitation effectively. Dopamine-induced excitatory depression weakens both monosynaptic excitation and disynaptic inhibition, however, the influence on disynaptic connection is larger than monosynaptic connection, due to nonlinear property in action potential generation. Thus, total excitation during high frequency inputs become larger under dopamine application. 
the Schaffer-collateral pathway, and in addition receives another excitatory connection directly projecting from the entorhinal cortex, which is called the temporoammonic pathway (Cajal, 1911) (Figure 2A). Thus, area CA1 integrates two distinct kinds of information, one from the trisynaptic circuit carrying processed information of the entorhinal cortex via dentate gyrus and area CA3, and the other from the temporoammonic pathway that carries direct information from the entorhinal cortex. In contrast to the trisynaptic circuit whose origin is neurons in layer II of the entorhinal cortex, the temporoammonic pathway is a projection from neurons in layer III of the entorhinal cortex (Witter and Amaral, 2004). Recent in vivo recording study indicates significant differences in spatial information coding between neurons in layer II and III (Hafting et al., 2008). Thus, these two pathways, the Schaffer-collateral and the temporoammonic pathways, appear to carry distinct information to area CA1.

Several studies, indeed, support distinct behavioral roles of each pathway in animal learning. When the temporoammonic pathway is lesioned, precise spatial firing in area CA1 (Brun et al., 2008) and memory consolidation (Remondes and Schuman, 2004) are impaired. On the other hand, when Schaffer-collateral inputs to area CA1 are disrupted, although animals maintain place recognition memory, they show deficits in recall of remote spatial locations (Brun et al., 2002) and one-trial contextual learning (Nakashiba et al., 2008). Taken together, area CA1 serves a critical integrational role for hippocampal function, determining how the information from the entorhinal cortex should be selected and processed before output from the hippocampus.

In CA1 pyramidal neurons, the Schaffercollateral pathway makes synaptic contacts at proximal regions of the apical dendrites, but in contrast, the temporoammonic pathway makes synapses at the distal most region. Each pathway has distinct synaptic properties (Golding et al., 2002; Remondes and Schuman, 2003), and modulates the other pathway via nonlinear dendritic integration in pyramidal neurons or indirectly via interneurons (Ang et al., 2005; Dudman et al., 2007; Levy et al., 1998; Remondes and Schuman, 2002).

Then, howwilldopaminemodulateinformation

Frequency-dependent signal gating A process in which the input stimulation frequency determines the amount or direction of modulation in information transmission through neural circuits. integration of these two pathways? When dopamine is applied to hippocampal slices, dopamine selectively depresses temporoammonic-CA1 synapses (Otmakhova and Lisman, 1999), and furthermore, changes a frequency-dependent profile of signal transmissions in this pathway (Ito and Schuman, 2007) (Figure 2A). The significance of this frequency-dependent signal transmission is suggested in the temporoammonic pathway, because the entorhinal cortex, which is the origin of this pathway, shows both theta (4-12 Hz) and gamma (40-100 Hz) oscillatory activities in behaving animals (Chrobak et al. 2000). As such, the presence of dopamine will impose a selection bias for the receipt of information, encoded differentially with oscillatory activities in the entorhinal cortex. Thus, dopamineinduced change in frequency-dependent signal transmission is likely to have a significant impact on functional coupling between the entorhinal cortex and the hippocampus.

\section{FUTURE PERSPECTIVE}

We focused in this review on the significance of neuromodulator's function on changing a frequency-dependent profile of signal transmission. Frequency-dependent signal transmission is an intrinsic propery of neural circuits and further investigation of this property will be essential to understand the brain. However, as far as we know, relatively few studies have examined frequencydependent modulation in signal transmission. Studies using brain slices are likely to be appropriate for examining this property because of the relative ease in measuring the input-output relation by applying artificially controlled stimuli. We think that further understanding of frequencydependent signal gating will open up a new view of neuronal network architecture and will serve as an important link between slice studies and in vivo brain function.

Experiments based on conventional electrical stimulation, however, should be carefully interpreted when attempting to explain in vivo brain networks. For example, in typical in vitro physiology experiments, stimulation is applied to same axons repeatedly to mimic oscillatory activities. In vivo, however, neurons participating in oscillatory activities do not necessarily emit spikes at the same rate of the oscillatory frequency, but rather generate spikes that are phase-locked to some aspects of the oscillation (Laurent, 2002). These differences may change the impact of frequency-dependent transmission. For example, the spatial influence of signal modulation due to convergence and divergence of axonal inputs generating phase-locked activities will be different in vitro and in vivo. An improved means of network manipulation, which allows differential activation of individual neurons, will elucidate more precise control of frequency-dependent signal transmission in neuronal circuits. 
Although we specifically focused here on neuromodulator-mediated gating of oscillatory activities, the influence of neuromodulators on the regional interactions of the brain is not limited this process. For example, individual neurons differ in preferred oscillatory frequencies of membrane potentials owing to the type and distribution of ion channels in the membrane. (i.e. resonance property; Buzsaki and Draguhn, 2004; Giocomo et al., 2007; Hutcheon and Yarom, 2000). These resonant properties may differentially be controlled by neuromodulators via changing kinetics of voltage-sensitive channels. Such modulations are likely to influence the generation and receipt of oscillatory activities in each brain area. Thus, neuromodulators have many effects and can influence each step of frequencydependent information transfer among brain areas including the generation, transmission and receipt of oscillatory activities.

In spite of many observed correlations between oscillatory frequency and specific brain function in vivo (Buzsaki and Draguhn, 2004; Osipova et al., 2006; Palva and Palva, 2007), it is still an open question whether information can be differentially encoded or decoded in neuronal circuits based on each oscillatory frequency. An integrative approach, recording/imaging from both brain slices and behaving animals together with circuit manipulations, will be necessary to pursue this question.

Our exploration of dopamine-induced changes in the frequency-dependent profile of signal transmission at the temporoammonic pathway has provided insight into a neuromodulator's function in a neuronal network. This approach can be applied to other areas of the brain for studying neuromodulation.

\section{ACKNOWLEDGEMENTS}

We thank members of the Schuman laboratory for discussions. H.T. Ito is supported by the Nakajima foundation. E.M. Schuman is an Investigator of the Howard Hughes Medical Institute.

\section{REFERENCES}

Andersen, P., Bliss, T. V., Lomo, T. Olsen, L. I., and Skrede, K. K. (1969). Lamellar organization of hippocampal excitatory pathways. Acta Physiol. Scand. 76, 4A-5A.

Ang, C. W., Carlson, G. C., and Coulter, D. A. (2005). Hippocampal CA1 circuitry dynamically gates direct cortical inputs preferentially at theta frequencies. J. Neurosci. 25, 9567-9580.

Bjorklund, A., and Dunnett, S. B. (2007). Dopamine neuron systems in the brain: an update. Trends Neurosci. 30, 194-202.

Blumenfeld, B., Preminger, S., Sagi, D., and Tsodyks, M. (2006). Dynamics of memory representations in networks with novelty-facilitated synaptic plasticity. Neuron 52, 383-394.

Boling, W., Olivier, A., and Fabinyi, G. (2002). Historical contributions to the modern understanding of function in the central area. Neurosurgery 50, 1296-1309, discussion 1309-1210.

Brun, V. H., Leutgeb, S., Wu, H. Q., Schwarcz, R., Witter, M.P., Moser, E. I., and Moser, M. B. (2008). Impaired spatial representation in CA1 after lesion of direct input from entorhinal cortex. Neuron 57, 290-302.

Brun, V. H., Otnass, M. K., Molden, S., Steffenach, H. A., Witter, M. P., Moser, M. B., and Moser, E. I. (2002). Place cells and place recognition maintained by direct entorhinalhippocampal circuitry. Science 296, 2243-2246.
Buzsaki, G., and Draguhn, A. (2004). Neuronal oscillations in cortical networks. Science 304, 1926-1929.

Cajal, S. R. Y. (1911). Histologie du Systeme nerveux de l'hommes et des vertebres, Vol II. Paris, Maloine.

Chrobak, J. J., Lorincz, A., and Buzsaki, G. (2000). Physiological patterns in the hippocampo-entorhinal cortex system. Hippocampus 10, 457-465.

Dobrunz, L. E., and Stevens, C. F. (1997). Heterogeneity of release probability, facilitation, and depletion at central synapses. Neuron 18, 995-1008.

Dudman, J. T., Tsay, D., and Siegelbaum, S. A. (2007). A role for synaptic inputs at distal dendrites: instructive signals for hippocampal long-term plasticity. Neuron 56, 866-879.

El-Ghundi, M., Fletcher, P. J., Drago, J., Sibley, D. R., O'Dowd, B. F., and George, S. R. (1999). Spatial learning deficit in dopamine $\mathrm{D}(1)$ receptor knockout mice. Eur. J. Pharmacol. 383, 95-106.

Engel,A. K., Fries, P., and Singer,W. (2001). Dynamic predictions: oscillations and synchrony in top-down processing. Nat. Rev. Neurosci. 2, 704-716.

Gasbarri, A., Sulli, A., Innocenzi, R., Pacitti, C., and Brioni, J. D. (1996). Spatial memory impairment induced by lesion of the mesohippocampal dopaminergic system in the rat. Neuroscience 74, 1037-1044.

Gasbarri, A., Sulli, A., and Packard, M. G. (1997). The dopaminergic mesencephalic projections to the hippocampal formation in the rat. Prog. Neuropsychopharmacol. Biol. Psychiatry 21, 1-22.

Giocomo, L. M., Zilli, E. A., Fransen, E., and Hasselmo, M. E. (2007). Temporal frequency of subthreshold oscillations scales with entorhinal grid cell field spacing. Science 315, 1719-1722.

Golding, N.L., Staff, N.P., and Spruston, N (2002). Dendritic spikes as a mechanism for cooperative long-term potentiation. Nature 418, 326-331.

Hafting, T., Fyhn, M., Bonnevie, T., Moser, M. B., and Moser, E. I. (2008). Hippocampus-independent phase precession in entorhinal grid cells. Nature 453, 1248-1252.

Hasselmo, M.E.(1995). Neuromodulation and cortical function: modeling the physiological basis of behavior. Behav. Brain Res. 67, 1-27.

Heine, M., Groc, L., Frischknecht, R. Beique, J.C., Lounis, B., Rumbaugh, G., Huganir, R. L., Cognet, L., and Choquet, D. (2008). Surface mobility of postsynaptic AMPARs tunes synaptic transmission. Science 320 201-205.

Hikosaka, O. (2007). Basal ganglia mechanisms of reward-oriented eye movement. Ann. N. Y. Acad. Sci. 1104 229-249.

Hutcheon, B., and Yarom, Y. (2000). Resonance, oscillation and the intrinsic frequency preferences of neurons. Trends Neurosci. 23, 216-222.

Ito, H. T., and Schuman, E. M. (2007). Frequency-dependent gating of synaptic transmission and plasticity by dopamine. Front. Neural Circuits 1,1 .

Knight, R. (1996). Contribution of human hippocampal region to novelty detection. Nature 383, 256-259.

Kodama, T., Honda, Y., Watanabe, M., and Hikosaka, K. (2002). Release of neurotransmitters in the monkey frontal cortex is related to level of attention. Psychiatry Clin. Neurosci. 56, 341-342.

Koike-Tani, M., Kanda, T., Saitoh, N., Yamashita, T., and Takahashi, T. (2008). Involvement of AMPA receptor desensitization in short-term synaptic depression at the calyx of Held in developing rats. J. Physiol. 586, 2263-2275.

Laurent, G. (2002). Olfactory network dynamics and the coding of multidimensional signals. Nat. Rev. Neurosci. 3, 884-895.

Levy, W. B., Desmond, N. L., and Zhang, D. X. (1998). Perforant path activation modulates the induction of long-term potentiation of the schaffer collateral - hippocampal CA1 response: theoretical and experimental analyses. Learn. Mem. 4, 510-518.

Lisman, J. E., and Grace, A. A. (2005). The hippocampal-VTA loop: controlling the entry of information into longterm memory. Neuron 46, 703-713.

Mainen, Z. F., and Sejnowski, T. J. (1996). Influence of dendritic structure on firing pattern in model neocortical neurons. Nature 382, 363-366.

Markram, H., Gupta,A., Uziel,A., Wang,Y., and Tsodyks, M. (1998). Information 
processing with frequency-dependent synaptic connections. Neurobiol. Learn. Mem. 70, 101-112.

McClure, S. M., Daw, N. D., and Montague, P. R. (2003). A computational substrate for incentive salience. Trends Neurosci. 26, 423-428.

Mori, M., Abegg, M. H., Gahwiler, B. H., and Gerber, U. (2004). A frequencydependent switch from inhibition to excitation in a hippocampal unitary circuit. Nature 431, 453-456.

Nakashiba, T., Young, J.Z., McHugh, T. J., Buhl, D. L., and Tonegawa, S. (2008). Transgenic inhibition of synaptic transmission reveals role of CA3 output in hippocampal learning. Science 319, 1260-1264.

Osipova,D., Takashima,A., Oostenveld, R., Fernandez, G., Maris, E., and Jensen, O. (2006). Theta and gamma oscillations predict encoding and retrieval of declarative memory. J. Neurosci.26, 7523-7531.

Otmakhova, N. A., and Lisman, J. E. (1999). Dopamine selectively inhibits the direct cortical pathway to the CA1 hippocampal region. J. Neurosci. 19, 1437-1445.

Palva, S., and Palva, J. M. (2007). New vistas for alpha-frequency band oscillations. Trends Neurosci. 30, 150-158.
Passingham, R. E., Stephan, K. E., and Kotter, R. (2002). The anatomical basis of functional localization in the cortex. Nat. Rev. Neurosci. 3, 606-616.

Pouille, F., and Scanziani, M. (2004). Routing of spike series by dynamic circuits in the hippocampus. Nature 429, 717-723.

Remondes, M., and Schuman, E. M. (2002). Direct cortical input modulates plasticity and spiking in CA1 pyramidal neurons. Nature 416 , 736-740.

Remondes, M., and Schuman, E. M. (2003). Molecular mechanisms contributing to long-lasting synaptic plasticity at the temporoammonic-CA1 synapse. Learn. Mem. 10, 247-252.

Remondes, M., and Schuman, E. M. (2004). Role for a cortical input to hippocampal area CAl in the consolidation of a long-term memory. Nature 431, 699-703.

Robbins, T. W. (2005). Chemistry of the mind: neurochemical modulation of prefrontal cortical function. J. Comp. Neurol. 493, 140-146.

Rutishauser, U., Mamelak, A. N., and Schuman, E. M. (2006). Single-trial learning of novel stimuli by individual neurons of the human hippocampus-amygdala complex. Neuron 49 , 805-813.
Schultz,W.(1998).Predictive reward signal of dopamine neurons. J. Neurophysiol. $80,1-27$.

Shepherd, G. M. (2004). The Synaptic Organization of the Brain. Oxford, UK: Oxford University Press.

Siegel, S. J., Agranoff, B. W., Albers, R. W. Fisher, S. K., and Uhler, M. D. (1999). Basic Neuochemistry. Philadelphia, PA: Lippincott Williams \& Wilkins.

Squire, L. R., Stark, C. E., and Clark, R. E (2004). The medial temporal lobe. Annu. Rev. Neurosci. 27, 279-306.

Takakusaki, K., Saitoh, K., Miyokawa, N., and Koyama,Y.(2006). Neurobiological basis of state-dependent control of motor behaviors. Sleep Biol. Rhythms 4, 87-104.

Varela, F., Lachaux, J.P., Rodriguez, E., and Martinerie, J. (2001). The brainweb: phase synchronization and largescale integration. Nat. Rev. Neurosci. 2, 229-239.

Venton, B. J., Zhang, H., Garris, P. A. Phillips, P. E., Sulzer, D., and Wightman, R. M. (2003). Real-time decoding of dopamine concentration changes in the caudate-putamen during tonic and phasic firing. $J$. Neurochem. 87, 1284-1295.

Wise, R. A. (2004). Dopamine, learning and motivation. Nat. Rev. Neurosci. 5, 483-494.
Witter, M. P., and Amaral, D. G. (2004). The Rat Nervous System. Amsterdam, Netherland: Elsevier Academic Press.

Zoli, M., Torri, C., Ferrari, R., Jansson, A., Zini, I., Fuxe, K., and Agnati, L. F. (1998). The emergence of the volume transmission concept. Brain Res. Brain Res. Rev. 26, 136-147.

Zucker, R. S., and Regehr, W. G. (2002). Short-term synaptic plasticity. Annu. Rev. Physiol. 64, 355-405.

Conflict of Interest Statement: The authors declare that the research was conducted in the absence of any commercial or financial relationships that could be construed as a potential conflict of interest.

Received: 19 September 2008; accepted: 04 November 2008; published: 15 December 2008.

Citation: Front. Neurosci. (2008) 2, 2: 138 144. doi: 10.3389/neuro.01.027.2008 Copyright: (c) 2008 Ito and Schuman. This is an open-access article subject to an exclusive license agreement between the authors and the Frontiers Research Foundation, which permits unrestricted use, distribution, and reproduction in any medium, provided the original authors and source are credited. 